\title{
The domain-specific and domain-general relationships of visuospatial working memory to reasoning ability
}

\author{
Zach Shipstead $^{1} \cdot$ Jade Yonehiro $^{1}$
}

Published online: 14 March 2016

(C) Psychonomic Society, Inc. 2016

\begin{abstract}
The degree to which visuospatial working memory (VSWM) is separable from working memory in general is an open question. On one hand, the construct is often researched as a unitary, domain-specific system. On the other, there is evidence that VWSM shares a common processing component with verbal memory. One might interpret this shared component as domain-general attention. We used confirmatory factor analysis to demonstrate that VSWM shares a domain-general component with verbal memory tasks and has a domainspecific component that is independent of verbal memory. Furthermore, the domain-general component was found to correlate with reasoning ability in both the visuospatial and verbal domains. The domain-specific component only correlated with reasoning ability when the tests had a strong visuospatial component. We argue that theories of VSWM need to place greater emphasis on its multiply determined nature.
\end{abstract}

Keywords Working memory capacity $\cdot$ Reasoning . Fluid intelligence $\cdot$ Visuospatial working memory

Working memory is a cognitive system that allows people to maintain and use goal-relevant information. The most influential model of working memory (Baddeley, 1986) divides it into three components: verbal and visuospatial short-term memory, as well a central executive attention that organizes information between the modalities.

Zach Shipstead

zach.shipstead@asu.edu

1 Department of Social and Behavioral Sciences, Arizona State University, Glendale, AZ 85306-4908, USA
An alternative perspective of working memory hypothesizes that there are actually two distinct working memory systems (Shah \& Miyake, 1996): One handles verbal information, and the other visuospatial, each with its own processing resources. Shah and Miyake demonstrated that performance on verbal working memory tasks predicts performance on tasks that involve reasoning with verbal materials, but not tasks that require reasoning with visuospatial materials. Likewise, visuospatial working memory (VSWM) tasks only predicted reasoning ability in the visuospatial dimension.

Although this finding is controversial (see Kane et al., 2004), the concept of a distinct VSWM has entered into mainstream psychology, ${ }^{1}$ bringing its own set of research questions, separate from the general study of working memory (see Luck \& Vogel, 2013). These questions include whether VSWM is limited by a discrete number of slots or is a flexible resource (Awh, Barton, \& Vogel, 2007; Bays \& Husain, 2008), whether VSWM stores features or integrated objects (Luck \& Vogel, 1997; Wheeler \& Treisman, 2002), and whether the fidelity or quantity of representations is more important to complex cognition (Fukuda et al., 2010). Recently, researchers have also begun to question whether individual differences in VSWM require an assumption of individual differences in storage capacity, or might be accounted for via attentional stability (Adam, Mance, Fukuda, \& Vogel, 2015; Fukuda, Woodman, \& Vogel, 2015; Shipstead, Harrison, \& Engle, 2015).

These are "either/or" questions and imply the existence of a distinct, unitary memory system. Asking whether the capacity of VSWM is bound by "discrete slots" or is a "flexible resource" assumes that VSWM is strictly subject to

\footnotetext{
${ }^{1}$ In particular, we refer to research using the visual-arrays task, which is described in the Method section.
} 
one capacity limitation. Yet, in the more traditional perspective of working memory (Baddeley, 1986), VSWM has at least two capacity limitations: domain-specific visuospatial storage and domain-general executive attention.

There is evidence that this domain-general resource is reflected in the performance of VSWM tasks. For instance, although the measurable capacity of VSWM is unaffected by simultaneous rehearsal of small amounts of verbal information (about two items; Luck \& Vogel, 1997; Morey \& Cowan, 2004), remembering larger amounts verbal information (about six items) does cause impairment (Morey \& Cowan, 2004; Saults \& Cowan, 2007). In essence, committing attentional resources to maintaining verbal information can reduce the storage capacity of VSWM. Moreover, individual differences in VSWM capacity predict a person's ability to retrieve verbal information from long-term memory (Shipstead, Lindsey, Marshall, \& Engle, 2014). VSWM capacity seems to reflect an executive resource that is important for remembering information in more than one memory domain (e.g., executive attention; Baddeley 1986; Engle, 2002).

\section{Present study}

We explored a two-component conceptualization of VSWM by examining the correlations between VSWM and simple verbal maintenance, as well as the correlation between VSWM and reasoning - in both the verbal and visuospatial dimensions. If VSWM includes both domain-specific (visuospatial storage) and domaingeneral (executive attention) components, then two important predictions can be made.

First, the presence of a domain-general resource assumes that individual differences in VSWM and verbal memory capacity are strongly correlated. If VSWM is unrelated to verbal memory, or if the correlation is small, this would solidly favor a distinct VSWM (e.g., Shah \& Miyake, 1996).

Second, if VSWM and verbal task performance share a common component, any variance that is common to VSWM and verbal memory task performance will consistently correlate to tests of reasoning ability. This will occur regardless of whether reasoning is defined using verbal or visuospatial tasks.

The two-component explanation of VSWM predicts that there will also be a domain-specific component of VSWM. This gives rise to a critical prediction. Although the domain-general factor will always be related to reasoning abilities, the domain-specific component will only relate to reasoning tasks that have a strong visuospatial component (see Kane et al., 2004). The domain-specific component of VSWM would only support task performance in the visuospatial domain.

\section{Method}

\section{Data collection and participants}

We examined the data via confirmatory factor analysis. Our predictors were expected to have strong correlations to our criterion variables. We therefore chose to have a moderate sample size of $150-170$ prior to beginning the study (Kline, 1998). Our stopping rule was to cease data collection at the end of the semester, provided we were within this range.

In all, 166 students were recruited from the Arizona State University subject pool. Six were excluded from the final analysis for being outside of our age range (18-35). One other was removed for refusing to follow instructions. This resulted in a final sample of 159. Their mean age was 22.98 years $(S D=3.90)$; 112 were female, 47 were male. All were compensated with credit toward course requirements.

\section{Tasks}

Participants were allotted $2 \mathrm{~h}$ to complete ten tasks. Three of the tasks measured VSWM, three measured verbal memory, and four measured reasoning ability (two spatial/two verbal).

VSWM All of the VSWM tasks were variants of the visualarrays tasks. Full descriptions of these tasks are available in Shipstead et al. (2014). In short, test takers were presented with an array of items that briefly disappeared (retention interval), then returned. The test taker's job was to indicate whether one object had changed relative to the first presentation. In these tasks, the arrays were presented for $250 \mathrm{~ms}$, followed by a $900-m s$ retention interval. Changes occurred on half of all trials. We selected $k$, which is an estimate of storage capacity, as the dependent variable for each task.

Three tasks were used: VA color, VA orient, and VA select. For VA color, the arrays were composed of four, six, or eight colored boxes (white, black, red, yellow, green blue, and purple), and the test takers decided whether a circled item had changed color. For VA orient, the arrays were composed of five or seven colored bars (blue or red) that were horizontal, vertical, or tilted $45^{\circ}$, and test takers needed to decide whether any item in the test array had changed orientation. For VA select, each trial began with the word "red" or "blue" (200 ms), indicating that test takers should only attend to red or blue items on that trial. Next, an array of either 10 or 14 bars at different orientations were displayed for $250 \mathrm{~ms}$; half of the bars were blue, and half were red. Memory was probed by superimposing a white dot on one of the bars, and test takers needed to decide whether this item had changed orientation. 
Verbal memory Verbal memory was measured using word span, letter span, and the primary memory portion of a free recall task. These tasks were chosen specifically because of their relationship to simple verbal maintenance capacity.

For the word span task, participants saw lists of words that needed to be recalled in the correct order. Each list was between two and seven items long, and each list length was presented twice in a session. The order was randomized, with words randomly drawn from a pool of 15 and presented at a rate of one per second. Responses were provided via mouseclick. The dependent variable was the total number of words recalled in the correct order for lists that were recalled correctly in their entirety.

For the letter span task, participants needed to remember a list of up to nine letters, randomly drawn from a limited pool of nine. This task began with a list of two letters. After three such trials, the lists were lengthened by one item, provided that the participant could correctly recall two of the three lists. Testing ended when either this could not be accomplished or the task had maxed out at list length nine. Letters were presented at a rate of four per second, and responses were provided via mouse-click. The dependent variable was the total number of lists correctly recalled.

In the free recall task, test takers saw ten words at the rate of one word per second. They were then allowed $30 \mathrm{~s}$ to type as many words as they could remember, in any order. Ten trials were run. The dependent variable was the primary memory component, as described by Tulving and Colotla (1970), which approximates the recency portion of the serial position curve.

Visuospatial reasoning Visuospatial reasoning was measured using matrix-reasoning and paper-folding tasks. These tasks approximated Raven's Progressive Matrices (Raven, 1990) and paper folding (Ekstrom, French, Harman, \& Dermen, 1976), but used newly developed, nonproprietary problems.

In matrix reasoning, eight shapes were arranged in a $3 \times 3$ matrix. The ninth location was blank. Test takers chose which of eight options would complete the series. They were allotted $5 \mathrm{~min}$ to complete ten problems. In paper folding, the test takers saw a depiction of a sheet of paper that was folded several times and then hole-punched. They were required to choose (from among five options) how the sheet would look if it were unfolded. Participants were again allowed $5 \mathrm{~min}$ to complete ten problems.

Verbal reasoning As an example of the nonsense syllogisms (Ekstrom et al., 1976), test takers were presented with "All turnips are dolphins. All dolphins are purple. Therefore, all turnips are purple." They were to determine whether the logic was sound and click a box labeled Good
Reasoning or another labeled Poor Reasoning. The dependent variable was the number of syllogisms correctly labeled in 5 min ( 15 possible).

The analogies (Berger, Gupta, Berger, \& Skinner, 1990) were " $A$ is to $B$ as $C$ is to " problems. Test takers selected the answer out of five response options that best completed each analogy. The dependent variable was the number of analogies correctly solved in $5 \min$ (12 possible).

\section{Results}

All correlations and descriptive statistics are available in Table 1. The reliabilities for the matrix problems and nonsense syllogisms were low. In the context of our analyses, this limits these tasks' ability to correlate with other tasks and to contribute to factors. However, as will be seen, the results are quite coherent.

The correlations were examined using confirmatory factor analysis (CFA). In this technique, performance similarities that people show across several tasks (e.g., verbal tasks) are extracted to form a latent factor (see the subsequent figures). Extraction of latent factors allows us to examine specific influences underlying the correlation matrix.

Fit statistics for each CFA are available on Table 2, where the abbreviations below are defined. In short, $\chi^{2} / d f$ should be less than 2. It is preferable that the RMSEA and SRMR be below .05 , but up to .10 is acceptable. The NNFI and CFI should be above .90 , but above .95 is preferred. The AIC can used to estimate whether added complexity is offset by new explained variance. Lower values are preferred. Mardia's test of multivariate normality of the data was 2.34 . This statistic should be under 3 (for more information on these statistics, see Kline, 1998).

\section{Modeling VSWM}

Our first CFAs examined the relationships among our memory variables. The model in Fig. 1a loaded the verbal and VSWM tasks on separate factors. Despite obvious differences between the tasks (verbal vs. visuospatial; serial order vs. parallel presentation), verbal memory and VSWM had a reasonably strong correlation of .55. In other words, individual differences in verbal memory and VSWM shared $30 \%$ of their variance. Although verbal memory and VSWM are separable, the latent factors underlying the respective sets of tasks are related. This favors the presence of a domain-general component.

We next cross-loaded the VSWM tasks onto the same factor as the verbal tasks to form a domain-general working memory factor $\left(\mathrm{WM}_{\mathrm{DG}}\right.$; Fig. $\left.1 \mathrm{~b}\right)$. We found no correlation between $\mathrm{WM}_{\mathrm{DG}}$ and VSWM because all shared variance 
Table 1 Correlations and descriptive statistics

\begin{tabular}{|c|c|c|c|c|c|c|c|c|c|c|}
\hline & 1 & 2 & 3 & 4 & 5 & 6 & 7 & 8 & 9 & 10 \\
\hline \multicolumn{11}{|c|}{ Verbal Memory Tasks } \\
\hline 1. Word span & - & & & & & & & & & \\
\hline 2. Letter span & .43 & - & & & & & & & & \\
\hline 3. PMFR & .17 & .30 & - & & & & & & & \\
\hline \multicolumn{11}{|l|}{ VSWM Tasks } \\
\hline 4. VA color & .13 & .17 & .32 & - & & & & & & \\
\hline 5. VA orient & .14 & .15 & .19 & .36 & - & & & & & \\
\hline 6. VA select & .31 & .23 & .30 & .52 & .36 & - & & & & \\
\hline \multicolumn{11}{|l|}{ Reasoning Tasks } \\
\hline 7. Matrix & .26 & .22 & .29 & .40 & .22 & .43 & - & & & \\
\hline 8. Paper fold & .26 & .11 & .24 & .27 & .19 & .38 & .29 & - & & \\
\hline 9. Analogies & .40 & .29 & .28 & .24 & .22 & .35 & .37 & .45 & - & \\
\hline 10. Nonsense & .26 & .22 & .27 & .19 & .15 & .33 & .28 & .37 & .51 & - \\
\hline Mean & 28.76 & 10.70 & 25.77 & 3.84 & 3.09 & 1.89 & 6.45 & 7.09 & 5.18 & 9.42 \\
\hline$S D$ & 13.04 & 3.14 & 6.21 & 1.01 & 1.25 & 1.18 & 1.69 & 2.41 & 2.52 & 2.29 \\
\hline Min & 0.00 & 3.00 & 5.00 & 0.52 & -1.27 & -1.00 & 1.00 & 1.00 & 1.00 & 4.00 \\
\hline Max & 68.00 & 21.00 & 41.00 & 5.62 & 6.00 & 5.48 & 10.00 & 10.00 & 12.00 & 15.00 \\
\hline Skew & .30 & .16 & -.60 & -.79 & -.37 & -.14 & -.60 & -.65 & .36 & .06 \\
\hline Kurtosis & -.24 & .51 & .88 & .56 & .46 & -.41 & .24 & -.49 & -.47 & .03 \\
\hline Reliability & .66 & .83 & .78 & .74 & .76 & .76 & .49 & .75 & .68 & .51 \\
\hline
\end{tabular}

PMFR = primary memory, free recall. All reliabilities were calculated using Cronbach's $\alpha$. Correlations above .17 are significant at $p=.05$.

was assigned to $\mathrm{WM}_{\mathrm{DG}}$, thus making VSWM domain-specific. In our models, this is denoted by a * next to VSWM.

Considering that the verbal tasks were chosen to be as basic and different from the VSWM tasks as possible, it is meaningful that this model converged and is coherent. There is very little, if any, task-specific variance between these sets of tasks. Any relationship suggests that a fairly general latent process is being measured.

The fit statistics for these models were reasonable, but not optimal (Table 2). However, as will be seen, the displayed factors work quite well in later models. Thus, we saw no need for alterations. ${ }^{2}$

\section{Modeling reasoning ability}

We next examined the relationships among the reasoning tasks. The first model (Fig. 2a) loaded visuospatial and verbal reasoning tasks onto the same factor, and the fit was exceptional (Table 2). Note that this factor is made up of variance

\footnotetext{
${ }^{2}$ Note that we did attempt a three-factor model with a verbal factor, but it did not converge. The demands of the verbal memory tasks were quite varied. This likely increased reliance on domain-general resources (rather than task-specific strategies), relative to the tasks used in studies such as Kane et al. (2004).
}

that is common to visuospatial and verbal reasoning tasks, and dimension-specific biases are minimized.

The second model divided the visuospatial and verbal reasoning tasks (Fig. 2b). REA $\mathrm{vs}_{\mathrm{v}}$ and $\mathrm{REA}_{\mathrm{verb}}$ (reasoning-visuospatial and reasoning-verbal, respectively) had an extremely strong correlation of .95 , which indicates that both factors reflect the same underlying reasoning processes. Any

Table 2 Fit statistics for all models

\begin{tabular}{lcccccccc}
\hline & \multicolumn{1}{c}{$\chi^{2}$} & $d f$ & $\chi^{2} / d f$ & RMSEA & SRMR & NNFI & CFI & AIC \\
\hline Figure 1a & 17.07 & 8 & 2.13 & .09 & .06 & .91 & .95 & 43.07 \\
Figure 1b & 15.22 & 6 & 2.54 & .10 & .06 & .88 & .95 & 45.22 \\
Figure 2a & 0.25 & 2 & 0.13 & .00 & .01 & 1.04 & 1.00 & 16.25 \\
Figure 2b & 0.07 & 1 & 0.04 & .00 & .00 & 1.04 & 1.00 & 18.07 \\
Figure 3a & 45.44 & 30 & 1.52 & .06 & .06 & .96 & .98 & 95.44 \\
Figure 3b & 22.28 & 15 & 1.49 & .06 & .05 & .96 & .98 & 64.28 \\
Figure 3c & 20.91 & 15 & 1.39 & .05 & .05 & .97 & .98 & 62.91 \\
Figure 4a & 47.24 & 32 & 1.48 & .06 & .06 & .97 & .98 & 93.24 \\
Figure 4b & 23.78 & 17 & 1.40 & .05 & .05 & .97 & .98 & 61.78 \\
Figure 4c & 23.14 & 17 & 1.36 & .05 & .05 & .97 & .98 & 61.14 \\
\hline
\end{tabular}

RMSEA = root-mean square error of approximation; SRMR = standardized root-mean square residual; $\mathrm{NNFI}=$ nonnormed fit index; $\mathrm{CFI}=$ cumulative fit index; AIC $=$ Akaike information criterion 
distinction between how these reasoning factors relate to our memory variables can be attributed to the test materials (visuospatial or verbal), but not to the presence or absence of reasoning in a factor. As well, the strong correlation indicates that splitting these tasks into two factors did not fundamentally disrupt the presence of domain-general reasoning.

\section{Full analyses}

Next, we tested the relationships of $\mathrm{WM}_{\mathrm{DG}}$ and VSWM to reasoning ability. Figure 3a presents the basic CFA. WM $\mathrm{DG}_{\mathrm{DG}}$ has a strong relationship to REA of .74. VSWM has a smaller, but still significant, relationship of .29 to REA. Thus, VSWM does relate to reasoning, above and beyond domain-general memory processes. The next two models tested the domain specificity of this correlation by introducing either a visuospatial or a verbal bias to REA.

The first test redefined REA as matrix reasoning and paper folding. ${ }^{3}$ Reasoning now had a strong visuospatial bias. The model in Fig. 3b indicates this had a substantial effect on the relationship of domain-specific VSWM to reasoning ability, with the correlation increasing to .62. Redefining reasoning using visuospatial tasks increased the importance of the domain-specific component of VSWM.

Next, we redefined REA as analogies and nonsense syllogisms. Reasoning now had a strong verbal bias. As is indicated in Fig. 3c, this also had a substantial effect on the domainspecific relationship of VSWM to reasoning, with the correlation dropping to a nonsignificant .14. When reasoning is defined using verbal reasoning tasks, the domain-specific component of VSWM is of little consequence to task performance.

Conversely, across these three models, the correlation of $\mathrm{WM}_{\mathrm{DG}}$ to reasoning was quite stable. As such, these models indicate that domain-general resources are important to reasoning ability, regardless of the dimension in which reasoning occurs (Kane et al., 2004). ${ }^{4}$

One may question the meaningfulness of the cross-loadings of VSWM on $\mathrm{WM}_{\mathrm{DG}}$, since most were small ( .3). Our final set of models (Fig. 4) present the relationship of VSWM to reasoning when these cross-loadings were omitted. Both domain-general and domain-specific variance was reflected by VSWM. The differences relative to Fig. 3 are stark. When VSWM is not cross-loaded on an executive attention factor, its correlation to $\mathrm{REA}_{\mathrm{vs}}$ is almost perfect (Fig. 4b). Additionally, when domain-general variance is present in VSWM, its correlation to $\mathrm{REA}_{\mathrm{verb}}$ is strong (Fig. 4c). Clearly, domain-general

\footnotetext{
${ }^{3}$ Due to the inclusion of paper folding, one might argue that this factor represents a type of visuospatial manipulation, instead of reasoning. However, the strong correlation of $\mathrm{REA}_{\mathrm{vs}}$ to REA $\mathrm{A}_{\mathrm{verb}}$ (Fig. 2b) contradicts this account; the only thing common to these factors is problem solving. ${ }^{4}$ See the Appendix for an alternative model that directly demonstrates a domain-specific relationship between VSWM* and visuospatial reasoning.
}

(a) Separate Memory Factors

(b) General/Domain Factors
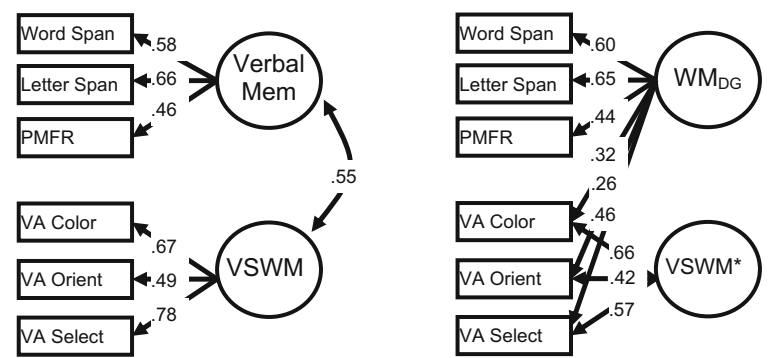

Fig. 1 Analyses examining the latent factors underlying memory task performance. PMFR = primary memory, free recall; VSWM = visuospatial working memory; VSWM* = visuospatial working memory with domain-general variance removed; $\mathrm{WM}_{\mathrm{DG}}=$ domaingeneral working memory capacity

resources are an important part of VSWM as it is currently measured in the psychological literature.

\section{Discussion}

VSWM represents at least two influences: domain-general working memory and domain-specific maintenance. Although this conclusion was predicted by Baddeley's (1986) classic model, it contrasts with perspectives that focus solely on domain-specific resources (e.g., Shah \& Miyake, 1996; Süß, Oberauer, Wittmann, Wilhelm, \& Schulze, 2002) or that assume that the properties of VSWM can be understood via either/or questions (e.g., slots OR resources; features OR objects; quality OR quantity; see Luck \& Vogel, 2013). VSWM is multiply determined, and its properties are likely more complex than the unitary-resource proposals that are common in the literature (e.g., Luck \& Vogel, 2013; Shah \& Miyake, 1996; Shipstead et al., 2015).

\section{VSWM and reasoning}

VSWM has a particularly strong relationship to reasoning ability, relative to verbal memory (Kane et al., 2004). This has been interpreted as evidence that VSWM is particularly important to human reasoning, and perhaps not separable from working memory in general (Klingberg, 2009). However, the present results suggest a more nuanced relationship. (a) One Reasoning Factor

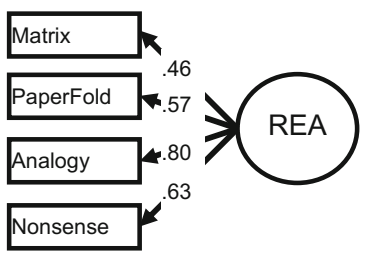

(b) Two Reasoning Factors

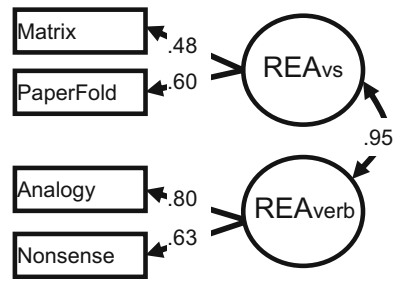

Fig. 2 Analyses examining the latent factors underlying reasoning task performance. REA = reasoning; vs = visuospatial; verb = verbal 
(a) Full CFA

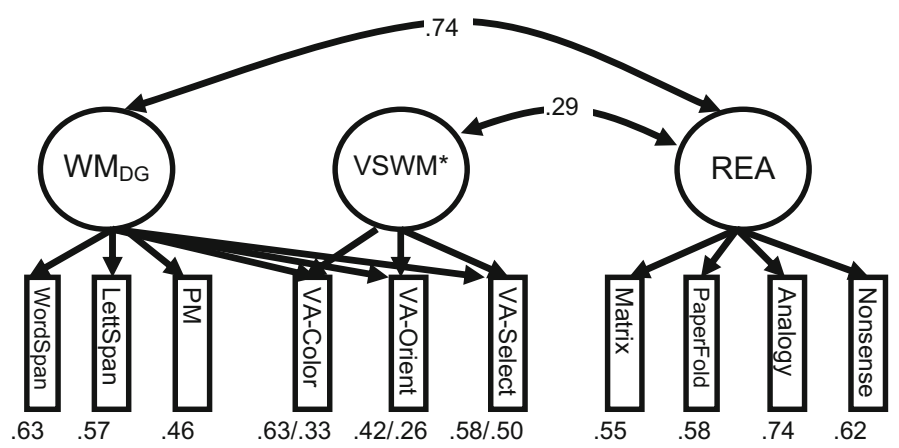

(b) Reasoning defined visuospatially

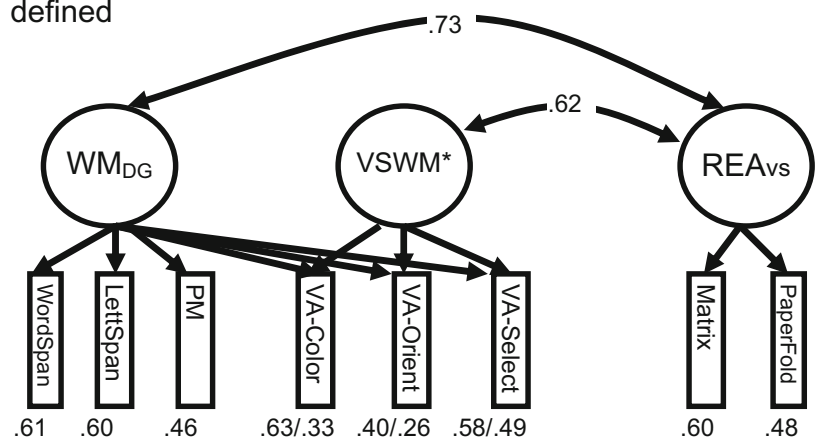

(c) Reasoning defined verbally

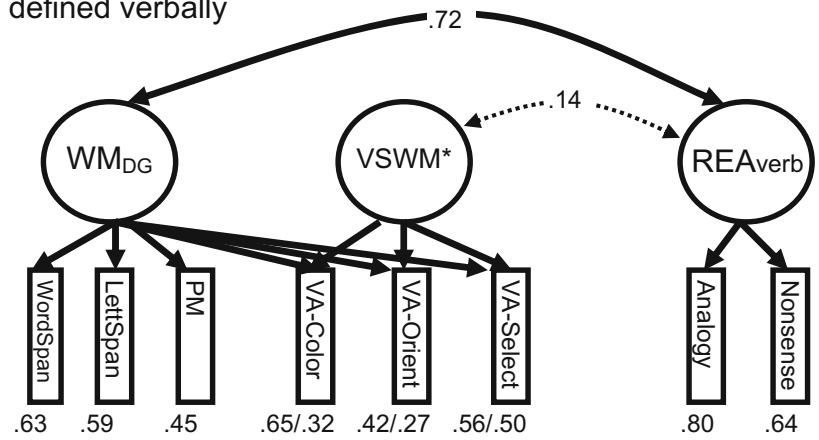

Fig. 3 Confirmatory factor analyses (CFAs) in which fluid intelligence was defined (a) through a balanced set of reasoning tasks, (b) visuospatially, or (c) verbally. Numbers under each task represent the factor loadings. For VSWM, numbers to the right of the slash are the

VSWM includes a domain-general component that relates to reasoning, regardless of the domain in which the reasoning tasks are presented ( $\mathrm{WM}_{\mathrm{DG}} \leftrightarrow$ REA; Fig. 3a-c). It also contains a domain-specific relationship that becomes more important as tests of reasoning become more visuospatially biased. We do not interpret this domainspecific component as reflecting reasoning itself. Instead, it reflects the components of VSWM that allow specific problems to be represented in memory. The domaingeneral component, on the other hand, has a strong relationship to reasoning abilities, regardless of the tasks that are used to measure reasoning. We view this component as representing active manipulation of information. Such processing is, however, supported by domain-specific memory. VSWM thus represents both general resources and the loadings on $\mathrm{WM}_{\mathrm{DG}}$. $\mathrm{WM}_{\mathrm{DG}}=$ domain-general working memory capacity; VSWM* = visuospatial working memory with domaingeneral variance removed; $\mathrm{REA}_{\mathrm{vs}}=$ visuospatial reasoning; $\mathrm{REA}_{\mathrm{verb}}=$ verbal reasoning

specific storage systems that are necessary to many types of reasoning task (e.g., matrix tasks).

\section{Domain-general variance as executive attention}

Although we interpret our domain-general factor as reflecting executive attention, another interpretation argues that it represents the focus of attention (Cowan, 2001). That is, the factor labeled $\mathrm{WM}_{\mathrm{DG}}$ represents domain-general storage. Although we have no issue with interpreting $\mathrm{WM}_{\mathrm{DG}}$ as representing focal attention, we argue that focal attention largely arises from the ability to stabilize attention on relevant information (Shipstead et al., 2015).

There is now ample evidence that tasks intended to measure VSWM do reflect such a stabilizing component (Fukuda, 
(a) Full CFA

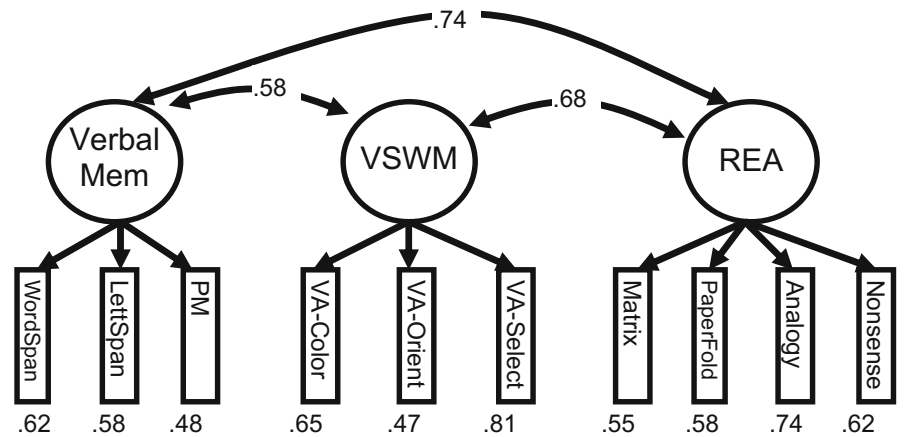

(b) Reasoning defined visuospatially

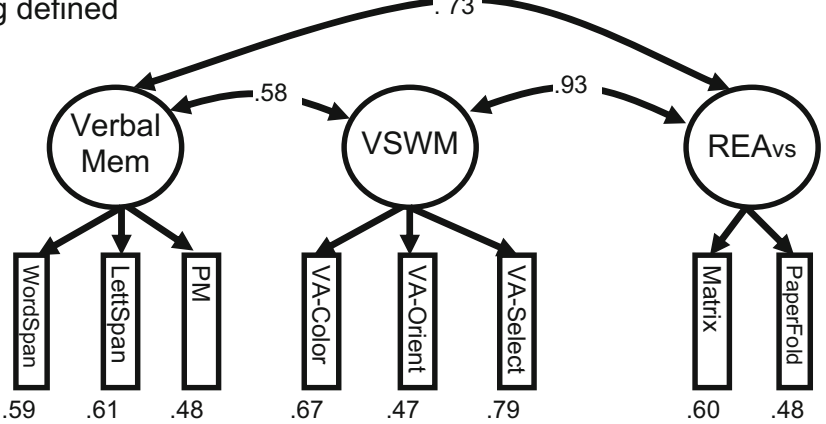

(c) Reasoning defined verbally

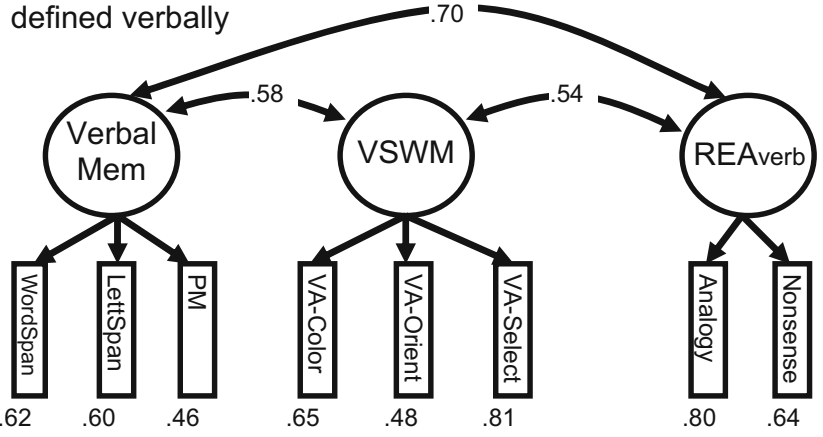

Fig. 4 Confirmatory factor analyses in which verbal memory and VSWM were treated as separate factors. Numbers under each task represent the factor loadings. VSWM = visuospatial working memory; $\mathrm{REA}_{\mathrm{vs}}=$ visuospatial reasoning; $\mathrm{REA}_{\mathrm{verb}}=$ verbal reasoning

Woodman, \& Vogel, 2015). Shipstead et al. (2015) recently demonstrated that VSWM factors, as presently defined, have a strong relationship to attention control. This relationship exists above and beyond more traditional measures of working memory capacity (e.g., complex span). Likewise, Fukuda and Vogel $(2009,2011)$ have repeatedly shown a relationship between VSWM and the ability to recover from distraction. Thus, we view the stabilizing effect of attention control as providing a probable account of the domain-general aspects of VSWM.

This relationship may seem surprising, since VSWM tasks do not always involve interruption or distraction; they simply require test takers to remember small amounts of information over a brief delay. There does not seem to be much need to engage attention control during task performance. Our interpretation is that information can be lost from the visuospatial storage system at many points. When items disappear from the screen, a test taker may mind wander, or be drawn to environmental distractions (e.g., random noises). Attentional engagement fluctuates on a trial-by-trial basis (Adam et al., 2015). Furthermore, when the probe array is displayed, it can have a destructive effect on the target memory (Makovski, Watson, Koutstaal, \& Jiang, 2010). In these cases, domain-general attention control allows test takers to stay focused on the tasks during delay periods and provides a mechanism to protect information from interference. This mechanism appears to be a common component of all valid tests of working memory capacity (regardless of domain), and it accounts for a large portion of their predictive validity across myriad contexts (Engle, 2002; Shipstead et al., 2015).

Author Note We thank Jeff Foster and Kenny Hicks for assistance with the matrix and paper-folding tasks. 


\section{Appendix: Model}

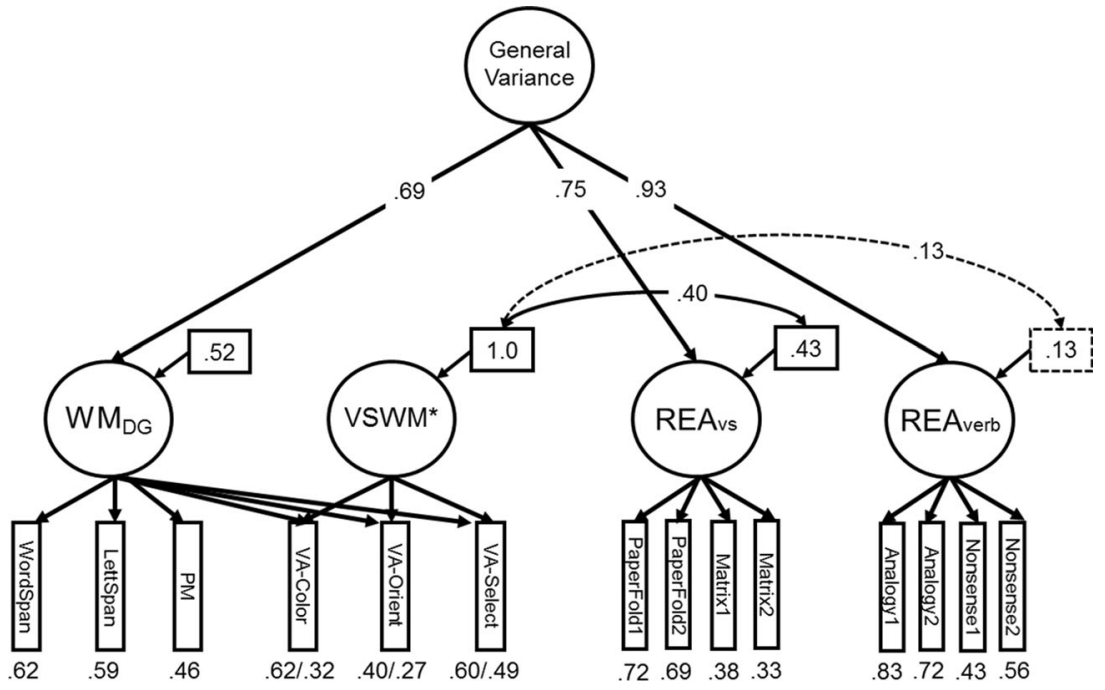

The factor labeled General Variance represents variance that is common to $\mathrm{WM}_{\mathrm{DG}}, \mathrm{REA}_{\mathrm{VS}}$, and $\mathrm{REA}_{\mathrm{verb}}$. The boxes next to each factor (disturbance factor) represent the proportions of variance in that factor that are not accounted for by General Variance. Correlations between the boxes represent correlations between the portions of the factor that are domain-specific. Although $\mathrm{REA}_{\mathrm{verb}}$ did not have significant domain-specific variance, an alternative model was run in which its loading on General Variance was constrained to be equal to that of $\mathrm{REA}_{\mathrm{vs}}$. This did create a significant disturbance factor, but the correlation to VSWM* remained nonsignificant (at .18; the correlation to $\mathrm{REA}_{\mathrm{vs}}$ remained significant, at .35). Convergence of this model was aided by dividing the reasoning scores in the first and second halves of all test items. $\chi^{2}=98.81, d f=69$, RMSEA $=.05$, SRMR $=.07, \mathrm{NNFI}=.92, \mathrm{CFI}=.94$

\section{References}

Adam, K. C. S., Mance, I., Fukuda, K., \& Vogel, E. K. (2015). The contribution of attentional lapses to individual differences in visual working memory capacity. Journal of Cognitive Neuroscience, 27, 1601-1616. doi:10.1162/jocn_a_00811

Awh, E., Barton, B., \& Vogel, E. K. (2007). Visual working memory represents a fixed number of items regardless of complexity. Psychological Science, 18, 622-628. doi:10.1111/j.1467-9280. 2007.01949.x

Baddeley, A. (1986). Working memory. Oxford, UK: Oxford University Press, Clarendon Press.

Bays, P. M., \& Husain, M. (2008). Dynamic shifts of limited working memory resources in human vision. Science, 321, 851-854. doi:10. 1126/science. 1158023

Berger, F. R., Gupta, W. B., Berger, R. M., \& Skinner, J. (1990). Air Force Officer Qualifying Test (AFOQT) form P: Test manual (AFHRL-TR-
89-56). Brooks Air Force Base, TX: Manpower and Personnel Division, Air Force Human Resources Laboratory.

Cowan, N. (2001). The magical number 4 in short-term memory: A reconsideration of mental storage capacity. Behavioral and Brain Sciences, 24, 87-185. doi:10.1017/S0140525X01003922

Ekstrom, R. B., French, J. W., Harman, M. H., \& Dermen, D. (1976). Manual for kit of factor-referenced cognitive tests. Princeton, NJ: Educational Testing Service.

Engle, R. W. (2002). Working memory capacity as executive attention. Current Directions in Psychological Science, 11, 19-23. doi:10. 1111/1467-8721.00160

Fukuda, K., \& Vogel, E. K. (2009). Human variation in overriding attentional capture. The Journal of Neuroscience, 29, 8726-8733. doi:10. 1523/JNEUROSCI.2145-09.2009

Fukuda, K., \& Vogel, E. K. (2011). Individual differences in recovery time from attentional capture. Psychological Science, 22, 361-368. doi: $10.1177 / 0956797611398493$

Fukuda, K., Vogel, E. K., Mayr, U., \& Awh, E. (2010). Quantity not quality: The relationship between fluid intelligence and working memory capacity. Psychonomic Bulletin \& Review, 17, 673-679. doi: $10.3758 / 17.5 .673$

Fukuda, K., Woodman, G. F., \& Vogel, E. K. (2015). Individual difference in visual working memory capacity: Contributions of attentional control to storage. In P. Jolicœur, C. Lefebvre, \& J. MartinezTrujillo (Eds.), Mechanisms of sensory working memory: Attention and performance XXV (pp. 105-119). San Diego, CA: Academic Press.

Kane, M. J., Hambrick, D. Z., Tuholski, S. W., Wilhelm, O., Payne, T. W., \& Engle, R. W. (2004). The generality of working memory capacity: A latent-variable approach to verbal and visuospatial memory span and reasoning. Journal of Experimental Psychology: General, 133, 189-217. doi:10.1037/0096-3445.133.2.189

Kline, R. B. (1998). Principles and practice of structural equation modeling. New York, NY: Guilford Press.

Klingberg, T. (2009). The overflowing brain: Information overload and the limits of working memory. New York, NY: Oxford University Press.

Luck, S. J., \& Vogel, E. K. (1997). The capacity of visual working memory for features and conjunctions. Nature, 390, 279-281. doi:10. $1038 / 36846$ 
Luck, S. J., \& Vogel, E. K. (2013). Visual working memory capacity: From psychophysics and neurobiology to individual differences. Trends in Cognitive Sciences, 17, 391-400. doi:10.1016/j.tics.2013.06.006

Makovski, T., Watson, L. M., Koutstaal, W., \& Jiang, Y. V. (2010). Method matters: Systematic effects of testing procedure on visual working memory sensitivity. Journal of Experimental Psychology: Learning, Memory, and Cognition, 36, 1466-1479. doi:10.1037/ a0020851

Morey, C. C., \& Cowan, N. (2004). When visual and verbal memories compete: Evidence of cross-domain limits in working memory. Psychonomic Bulletin \& Review, 11, 296-301. doi:10.3758/ BF03196573

Raven, J. C. (1990). Advanced progressive matrices. Oxford, UK: Oxford Psychologists Press.

Saults, J. S., \& Cowan, N. (2007). A central capacity limit to the simultaneous storage of visual and auditory arrays in working memory. Journal of Experimental Psychology: General, 136, 663-684. doi: 10.1037/0096-3445.136.4.663

Shah, P., \& Miyake, A. (1996). The separability of working memory resources for spatial thinking and language processing: An individual differences approach. Journal of Experimental Psychology: General, 125, 4-27. doi:10.1037/0096-3445.125. 1.4

Shipstead, Z., Harrison, T. L., \& Engle, R. W. (2015). Working memory capacity and the scope and control of attention. Attention, Perception, \& Psychophysics, 77, 1863-1880. doi:10.3758/ s13414-015-0899-0

Shipstead, Z., Lindsey, R. B., Marshall, R. L., \& Engle, R. W. (2014). The contributions of maintenance, retrieval and attention control to working memory capacity. Journal of Memory and Language, 72, $116-141$.

Süß, H.-M., Oberauer, K., Wittmann, W. W., Wilhelm, O., \& Schulze, R. (2002). Working-memory capacity explains reasoning ability — and a little bit more. Intelligence, 30, 261-288. doi:10.1016/S01602896(01)00100-3

Tulving, E., \& Colotla, V. A. (1970). Free recall of trilingual lists. Cognitive Psychology, 1, 86-98.

Wheeler, M. E., \& Treisman, A. M. (2002). Binding in short-term visual memory. Journal of Experimental Psychology: General, 131, 4864. doi:10.1037/0096-3445.131.1.48 